\title{
Postcoital Bleeding
}

National Cancer Institute

\section{Source}

National Cancer Institute. Postcoital Bleeding. NCI Thesaurus. Code C35220.

Non-menstrual bleeding that occurs during or after sexual intercourse. 